\title{
Verhaltenstherapie
}

\section{Band 15, Heft 2, Juni 2005}

Editorial

76 Von Freud zu Meaney: Die Zukunft der

Psychotherapie hat begonnen

Hellhammer, D. (Trier)

Originalarbeit

78 Ambulante vor- und nachbereitende Maßnahmen zur Optimierung der stationären psychosomatischen Rehabilitation - Ergebnisse des Bad Dürkheimer Prä-Post-Projekts Bischoff, C.; Gönner, S.; Ehrhardt, M.; Limbacher, K. (Bad Dürkheim)

Übersichtsarbeiten

88 Bibliotherapie

Grahlmann, K.; Linden, M. (Teltow/Berlin)

94 Biofeedback und zahnmedizinische Behandlungsansätze bei temporomandibulären Störungen und Bruxismus Korn, H.-J. (Prien)

103 Suizid im Krankenhaus - Überblick und aktueller Stand zum Suizid während stationärer psychiatrischer Behandlung

Wolfersdorf, M. (Bayreuth)

Interview

110 Michael Meaney: Wie die Zuwendung der Eltern die Stressvulnerabilität beeinflusst: Molekularbiologische Grundlagen sozialer Erfahrung Hellhammer, D. (Trier)

114 Neuigkeiten vom Buch- und Zeitschriftenmarkt

119 Fort- und Weiterbildung

124 Mitteilungen der Verbände

128 Tagungen und Kongresse

74 Impressum

132 Hinweise für Autoren

U2 Erläuterungen zum Titelbild (2. Umschlagseite) Leibl, C.; Breindl, K.; Naab, S. (Prien)
Vol. 15, Issue 2, June 2005

Editorial

76 From Freud to Meaney: The Future of Psychotherapy Has Begun Hellhammer, D. (Trier)

Original Article

78 Outpatient Preparation and Aftercare to Improve the Effects of Psychosomatic Inpatient

Rehabilitation

Bischoff, C.; Gönner, S.; Ehrhardt, M.; Limbacher, K. (Bad Dürkheim)

Review Articles

88 Bibliotherapy

Grahlmann, K.; Linden, M. (Teltow/Berlin)

94 Biofeedback in the Treatment of Temporomandibular Disorders and Bruxism Korn, H.-J. (Prien)

103 Inpatient Suicide in the Hospital - Overview and Recent Data on Suicide during Psychiatric Inpatient Treatment Wolfersdorf, M. (Bayreuth)

Interview

110 Michael Meaney: How Parental Care Affects Stress-Vulnerability: Biomolecular Underpinnings of Social Experience Hellhammer, D. (Trier)

114 Articles and Books

119 Education

124 Information by Behavior Therapy Associations

128 Meetings and Conferences

74 Imprint

132 Guidelines for Authors

C2 Explanations on the Title (Inside front cover) Leibl, C.; Breindl, K.; Naab, S. (Prien) 